\title{
Congenital plasmodium vivax malaria mimicking neonatal sepsis: a case report
}

\author{
Jain $\mathbf{A}^{1}$, Saraswat $\mathbf{D}^{2}$ \\ ${ }^{1}$ Dr Anil Jain, Professor Pediatrics, JLN Medical College, Ajmer, Rajasthan, ${ }^{2}$ Dr. Devina Saraswat, Post graduate \\ Resident, Pediatrics, JLN, Medical College, Ajmer, Rajasthan, India
}

Address for Correspondence: Dr. Devina Saraswat, Email: drdevinasaraswatdevina060788@ gmail.com

\begin{abstract}
A case of congenital malaria by plasmodium vivax initially mistaken for neonatal sepsis is described. Correct diagnosis requires high index of suspicion with inclusion of congenital malaria in the differential diagnosis of neonatal sepsis even if the mother has no proven malaria episodes during gestational period.
\end{abstract}

Keywords: Congenital Malaria, Differential Diagnosis of Sepsis, Maternal History of Malaria

\section{Introduction}

Severe parasitization of the placenta by malaria parasite particularly P.Falciparum, P.Vivax also called as Placental Malaria can result in transplacental transmission of parasite to fetus and consequently congenital malaria [1,2-4].

However it is pertinent to state that many of the reports presenting data on the burden of malaria among young children appear not to focus on infant specifically. This is presumably because it is thought that infants under the age of six months are relatively protected against clinical malaria as a result of transfer of maternal antibodies and the presence of fetal haemoglobin.

Precise definition of congenital malaria is still a subject that is not devoid of controversy, but symptoms usually occur 10 to 30 days postpartum. The disease can be observed in a day old baby or be delayed for weeks or months[1]. Studies had speculated [2,1]- that effectiveness of the placenta to restrain the malaria parasite passage to the fetus and the remarkable capacity of the fetus to resist infection $[2,3,4,5]$ was responsible for rarity of congenital malaria. It is pertinent to state that most cases of congenital malaria are misdiagnosed initially because of lack of specific symptoms and general awareness of this uncommon disease [6].

Congenital malaria was defined as the presence of asexual stages of malarial parasite in cord blood smear at delivery or peripheral blood smear of the baby in the first 7 days of life, irrespective of clinical symptoms $[7,8]$.

Therefore for purpose of performing accurate diagnosis of congenital malaria a good index of suspicion, a careful physical examination and peripheral blood smear are therefore needed[4,5,6,8]. Sometimes parasitemia cannot be shown on blood smear and plasmodial antigen detection or PCR of blood may be necessary[8].

\section{Case Presentation}

A 18 day old male born to gravida 1 parity 1 hindu female resident of Nagaur, Rajasthan was admitted in department of neonatology,NICU, JLN Ajmer for intermittent fever of 4 days duration with temperature 40 degree celcius . The mother had a spontaneous normal delivery at 40 week gestation and no abnormal events during pregnancy period. The newborn birth weight was $3.2 \mathrm{~kg}$ was on exclusive breastfeeding.

Manuscript received: $20^{\text {th }}$ Oct 2015

Reviewed: $5^{\text {th }}$ Nov 2015

Author Corrected: $12^{\text {th }}$ Nov 2015

Accepted for Publication: 24 ${ }^{\text {th }}$ Nov 2015 
On admission the infant was febrile, with excessive cry and refusal to feed. Physical examination showed pallor, hepatomegaly ( $3 \mathrm{~cm}$ below costal margin), splenomegaly( $4 \mathrm{~cm}$ below costal margin). Respiratory and cardiovascular systems were normal.
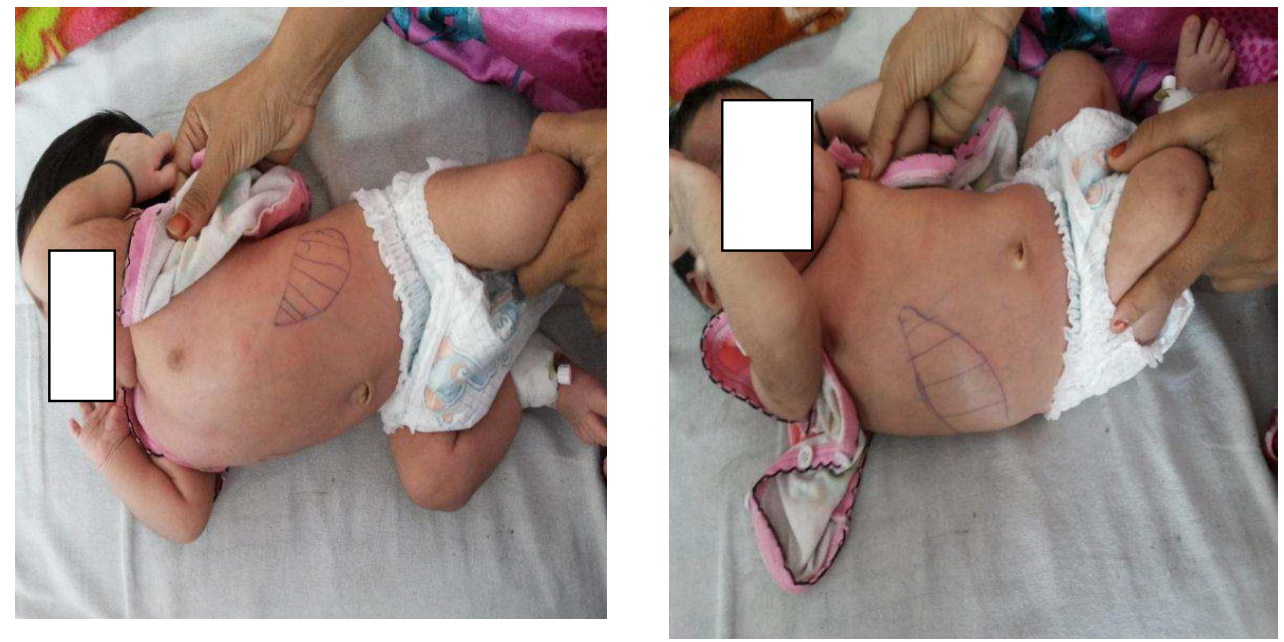

Fig 1 \& 2: Baby Showing Hepato-Splenomegaly

Laboratory evaluation - mild anaemia ( $\mathrm{Hb} 11 \mathrm{~g} / \mathrm{dl})$ normal total $\mathrm{RBC}(3,00,000 / \mathrm{cu} . \mathrm{mm})$ and WBC $(7,000 / \mathrm{cu} \mathrm{mm})$ platelets $(1,00,000 / \mathrm{cu} \mathrm{mm}), \mathrm{CRP}$ reactive, $\mathrm{SGOT}=72, \mathrm{SGPT}=100, \mathrm{~S}$. Bilirubin=2.1). The remaining biochemistry parameters and metabolic values were within normal limits.A clinical diagnosis of neonatal sepsis was done and I.V antibiotic cefotaxime,amikacin were started.
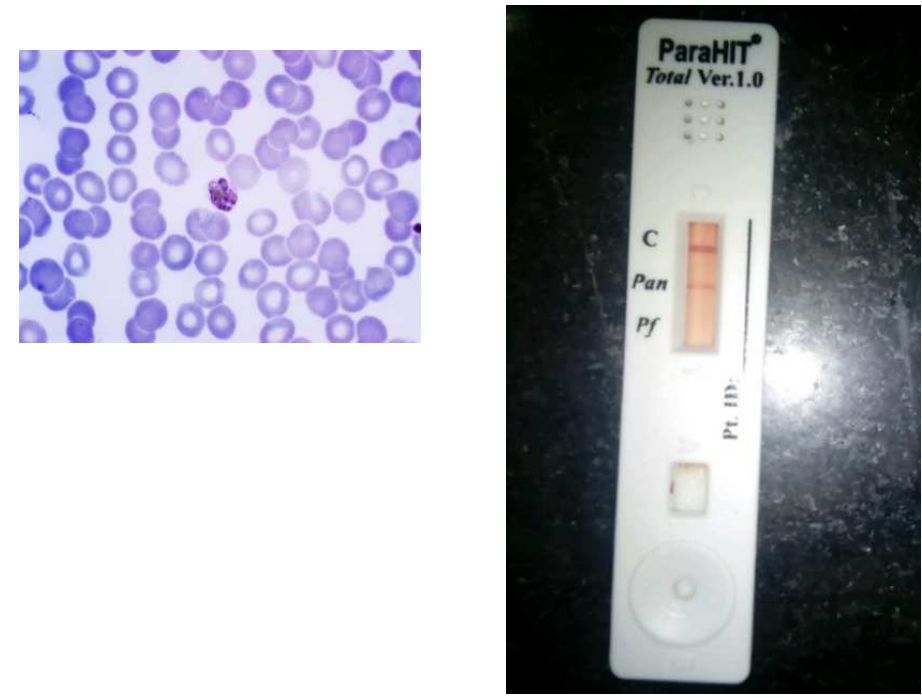

Fig 3 \& 4: Peripheral smear \& slide test

After 24 hours of admission a PBF was sent along M.P Card test, reports revealed P.Vivax positive and PBF showed P. Vivax trophozoites, few schizonts. Hence diagnosis of P.Vivax was confirmed.[8,10,12,13].

The infant was administered oral chloroquine $10 \mathrm{mg} / \mathrm{kg}$ first day and $5 \mathrm{mg} / \mathrm{kg}$ for next 4 days. Five days later parasitemia cleared completely, as suggested by negative card test and PBF.WBC count increased to 13,000/cu mm, CRP non reactive.Infant was discharged on day $7^{\text {th }}$ with normal $\mathrm{HbValue,}$ platelet count, WBC ,RBC count and biochemical profile.

The history of mother was re-evaluated. She was a primigravida and told about high grade fever she had at 36 week pregnancy, she took local treatment for it. 
Fever was high grade associated with chills and rigor, every alternate day and relieved with medication, she was given single I.M injection by local a practitioner( probably artemether) no documents were available, no blood test were done. Now when mother PBF was examined it was negative for malaria parasite.

\section{Discussion}

This case showed that diagnosis of congenital malaria should be considered as an important differential of neonatal sepsis in infants who are born to mothers coming from malaria endemic countries with or without malaria disease during pregnancy and newborn with hepatospleenomegaly, thrombocytopenia and anaemia. $[4,5,6,9]$.

However proven history of malaria episodes of mother during gestational age is not essential, as many go undiagnosed [10,11]. Postulated Mechanism for congenital malaria include maternal transfusion of malarial parasite into fetal circulation either at the time of delivery or during pregnancy, direct penetration through chorionic villi, or penetration through premature separation of placenta.[2]

The remarkable capacity of fetus to resist infection has been demonstrated. This resistance can reflect the physical barrier of placenta or passive transfer of maternal antibodies, unfavourable environment offered by fetal RBC due to fetal $\mathrm{Hb}$ concentration[4,7,12]

It can be speculated that the mother had episode of $\mathrm{P}$. Vivax malaria during third trimester whichwas mild, resolved spontaneously and remained undiagnosed. Moreover Vivax malaria is not associated with miscarriage, stillbirth or premature delivery. $[2,6,10]$.

In this case mother presented with mild anaemia and infant was delivered spontaneously at term, normal birth weight. The time of onset of clinical symptoms in congenital malaria can vary immediately after birth to ten weeks age.[9,10,12]. Diagnosis is simple with microscope examination of blood films, with strong clinical suspicion.[7,8,13]. Clinical feature of congenital malaria include anaemia, fever, hepatosplenomegaly, poor feeding, lethargy, irritability, jaundice, severe thrombocytopenia with or without bleeding is also frequently seen.[4,5,6,13]. The treatment requires a blood schizonticide like chloroquine whereas primaquine is unnecessary as in congenital malaria there is no hepatic stage of parasite.[10,14].
The higher prevalence of congenital malaria by P.Vivax than falciparum is due to longer incubation period in Vivax and relatively milder clinical presentation which allows for more maternal episodes to go undiagnosed and untreated.a potential additional determinant is represented by contraindication of drugs that can eradicate liver stage of parasite during pregnancy thus increasing likelihood of late relapses[1].

\section{Conclusion}

In conclusion $\mathrm{P}$. Vivax congenital malaria is a rare condition that should be included in differential diagnosis of sepsis in newborn at epidemiological risk.[5,6] More studies are needed to be done to assess the mechanism of maternal transmission of P.Vivax .

Ethical approval: Obtained from Institutional Ethics Committee (Human Studies)

Funding: No funding sources.

Conflict of Interest: The authors declare no conflict of interest

\section{Reference}

1. Nosten F, Mcgready, R.Simpson JA, Thwai KL, Balkans, Chothei, HKirijaroen L, Looareesuwans S, White NJ: Effects of Plasmodium Vivax Malaria in Pregnancy. Lancet 1999,354;546-549.

2. Rogerson SJ, Hvjjd L, Duffy PE, Leke RFG, Taylor D: Malaria in Pregnancy :Pathogenesis \& immunity Lancet 2007, 7:105 -116.

3. Siriez JY, De Pontual, Poilane L, Ledeur F, Haouchine, Massine Lac: Congenital Malaria as result of Plasmodium Malarial in an infant born to HIV seropositive woman. Trop Med 2005,65: 477-81.

4. Ibhanesebhor SE: Clinical characteristic of neonatal malaria. J Tropical pediatr 2005,65:477:81

5. Voitier G, Arsac M, Farnoux C, Mariani- Kurkdjian $\mathrm{P}$, Baud O, Aujard Y: Congenital Malaria in neonates : two case report and review of literature. Paediatrica 2008, $97: 500-512$.

6. DeSilva DHG, Mendis KN, Premaratne UN, Jayatilleke SM D'Souza: Congenital Malaria due to P.Vivax : a case report from Sri Lanka. Trans R SOC Trop Med Hyg 1982 75:33-345. 
7. Miller IJ, Telford SR. Placental Malaria. Nengl J Med 1996, 335-98.

8. Wilson RJ, Pasvol G, Weatherall DJ. Invasion and Growth of Plasmodium falciparum in different types of human erythrocyte. Bull World Health organ 1977,55:179-186.

9. Lesko CR, Arguin PM, Newborn RD. Congenital Malaria in the United States. Arch PediatrAdolesc Med 2007, 161: 1062-1067.

10. Krause PJ: Malaria (plasmodium) Nelson Textbook Pediatrics WB Saunders Company. Philadelphia 16: 2000,174- 978.
11. AlAirshiHM, AlHifzilS, Ishag. Chloroquine resistant $\mathrm{P}$ falciparum Malaria in extremely premature infant. Ann Saudi Med 1999,19:245:247.

12. Fischer PR: Malaria and Newborns. J Trop Pediatrics 2003,49:132-134.

13. Baspinar O, Bayraktaroglu Z, KarsligilT, BayramA, CoscunY, A rare case of Anemia and Thrombocytopenia in a newborn: congenital Malaria. Turk JournPediatr 2006, 48:63- 658

14. Care of newborn, Meharbaan singh, Seventh edition,chapter 16, 211-214.

\section{How to cite this article?}

Jain A, Saraswat D. Congenital plasmodium vivax malaria mimicking neonatal sepsis: a case report. Pediatr Rev: Int J Pediatr Res 2015;2(4):160-163.doi:10.17511/ijpr.2015.i04.22 